\title{
Contents, Vol. 57, 1930
}

\section{Inhaltsverzeichnis.}

Or $1 / 8$ inalarbeiten.

SOite

Bauer, Hugo, Die Ausseheidung des Myosalvarsans 255

Bierbaum, K., und H. Gottron, Zur Kenntnis des Erysipeloids Rosen-bach unter besonderer

Berücksichtigung seiner Beziehungen zum

Schweinerotlauf

5

Curth, Wilhelm, Psoriasis und endokrines System. Untersuchungen

mittels der interferometrischen Methode nach Abderhalden-Hirsch 362 Fischl, Friedrich,

Übergangsformen zwischen Eczema seborrhoicum

und Pityriasis lichenoides chronica 56

Gottron, H., siehe Bierbaum, K.

Hoffmann, Erich, Entstehung, Wesen, Erkennung, Verhütung und

Frühheilung der angeborenen Syphilis (Lues innata s. connatalis). . 145 Jacobs, J., und K. H.

Voh winkel, Die Malariathcrapie der Früh-und

Spätlues. (Fünfjährige Erfahrungen der Essener Hautklinik) .... 321

Kadisch, Die Hitzeresistenz der Dermatophyten 412

Kumer, L., Über Haut- und Mundschleimhauterscheinungen beim Ulcus

vulvae acutum , $\quad 401$

Löhe, H., und H. Rosenfeld, Wismutpigmentierungen der Blasen-

schleimhaut. Erste Mitteilung, 250

Mallinckrodt-Haupt,Astavon, Milbenerkrankungen des Menschen

(Fortsetzung). Eiu neuer Trombidioseherd in der Eifel 191

Marcus, Karl, Beitrag zur Kenntnis der Prophylaxe bei Syphilis congenita 163

Mayr, Julius K., Die neue Dermatologische Klinik und Poliklinik der Universität München 366

Memmesheimer, Alois M., Der Frühjahrsgipfel des Ekzems und seine

Erklärungsmöglichkeiten. (Ein Beitrag zur Geographie der Hautkrankheiten) 27

Nathan, E., und Fr. Stern, Über den Mineralgehalt der Haut unter normalen und pathologischen Bedingungen. V. Mitteilung: Über den

Chlor- und Wassergehalt der normalen und experimentell durch Quarzbestrahlung entzündeten Kaninchenhaut 272

Oretschkin, E. S., siehe Perkel, J. D.

Parl, Helmut, Das Guttadiaphot in der Dermatologie419

Perkel, J. D., und E. S. Oretschkin, Zur Frage der Übertragung der

Syphilis auf die dritte Generation 47

Potaschnik, M. B., Über die Verwendung des essigsauren Thalliums 
bei Pilzerkrankungen 64

Potaschnik, M., siehe Wainstein, A.

Rosenfeld, H., siehe Löhe, H.

Schmidt, P. W., Über scrophulodermähnliche Brückenbildung, eine seltene Erscheinungsform bei Spätlues 347

IV Inhaltsverzeichnis.

Seite Schönfeld, W, Differentialdiagnostische klinische Bemerkungen zu

Trautwein, Karl, die Maul- und Klauenseuche-Infektion beim Men-

schen 248

Schubert, Martin, Versuche mit Ultraviolett durchlässigen Fenster-

gläsern 358

Stern, Fr., siehe Nathan, E.

Trautwein, Karl, Die Maul- und Klauenseuche-Infektion beim Men-

schen. (Hierzu Tafel I-III.) 241

Vohwinkel, K. fl., siehe Jacobs, J.

Wainstein, A., und M. Potaschnik, Die aktive Methode Tsü in der

Serodiagnose der Syphilis 201

Wegner, Erna, Die Erfolge der Behandlung der Lues innata .... 178 Winter, Hans, Blutgruppen in der Dermatologie; Blutgruppenver-

teilung in Oberhessen 432

Georg Arndt †

Gesellschaftsberichte.

Berliner Dermatologische Gesellschaft. Sitzung vom 9. VII. 1929

- $\quad$ Nachtrag zur Sitzung vom 11. VI. 1929

Kölner Dermatologische Gesellschaft. Sitzungen vom 31. V., 28. VI. und 93

26. VII. 1929 94

XVI. Kongreß der Deutschen Dermatologische n Gesellschaft in Königsberg i. Pr. Von Dr. Louis, Bonn 204

I. Internationaler Kongreß für Aktinologie vom 22. bis 29. Juli 1929 in

Paris. Von Philipp Keller, Freiburg i. Br

229

Royal Society of Medicine, Section of I? ermatology. Sitzungen vom

20. XII. 1928 und 17.1. 1929

96

Sitzung vom 21. II. 1929

278

North of England Dermatological Society. Erste Tagung in Manchester am 8. HI. 1929 100

Société Française de Dermatologie et de Syphiligraphie. Sitzungen vom

8. XL, 13. XII. 1928 und 10.1. 1929

Reunion Dermatologique de Nancy. Sitzung vom 19. XII. 1928

Periodische Literatur. Zusammengestellt von Dr. Arthur Alexander-

Charlottenburg $\quad 109,231,279,369,446$

Buchbesprechungen 142, 315, 395, 461

Personalien und Tagesnachrichten 144, 239, 319, 399, 461

Zur 50jährigen Wiederkehr der Entdeckung des Gonococcus durch Albert

Neíßer (Mai 1879) 399

Sachregister 463

$\mathrm{N}$ amen register $\quad 469$ 